\title{
Epidemiological characteristics and early complications after spinal cord injury in Former Yugoslav Republic of Macedonia
}

\author{
Vesna Miloshevska Jakimovska, ${ }^{1,2}$ Fin Biering-Sørensen ${ }^{3}$ Ingeborg Beate Lidal ${ }^{1}$ Emil Kostovski $\mathbb{C}^{1}$
}

Received: 18 February 2019 / Revised: 3 August 2019 / Accepted: 6 August 2019 / Published online: 19 August 2019

(c) The Author(s), under exclusive licence to International Spinal Cord Society 2019

\begin{abstract}
Study design Prospective cohort study

Objectives To describe epidemiological data and complications after acute traumatic spinal cord injury (tSCI) in Former Yugoslav Republic of Macedonia (FYROM).

Setting University Clinic for Traumatology, Orthopedics, Anesthesia and Intensive Care Unit and Emergency Center (TOARILUC), Mother Teresa, Skopje, FYROM.

Method During the inclusion period March 2015 to September 2016, 38 tSCI patients were included. MRI, CT scan, and clinical examinations including International Standards for Neurological Classification of SCI were performed at admission. The information included: demographic data, transport type, date of admission and discharge, past illnesses, addiction habits, cause and type of injury, injury level, associated injuries, injury-related complications, and mortality.

Results Mean age was 43 years (median 41, range 17-83). Seventeen patients had a complete and 15 an incomplete SCI, six were unknown. Most frequent causes for tSCI were traffic accidents (42\%) and falls (40\%), 24\% of the accidents were contracted at work. Sixteen patients were ventilator dependent at some point during the acute period. Common complications were pressure ulcers, gastrointestinal-related, and infections. Hospital length of stay (LOS) ranged from 1 to 73 days. The in-hospital mortality rate was $32 \%$.

Conclusion The annual incidence of tSCI in FYROM was in 2015-16 13 persons/million inhabitants per year. The epidemiological profile of tSCI in FYROM implies that preventive measures should be taken to reduce incidence of accidents in traffic and at work places. The high mortality rate and complications underline further actions to improve the acute care of tSCI in FYROM.
\end{abstract}

\section{Introduction}

The incidence of traumatic spinal cord injury (tSCI) vary across the world, assorted literature describes from 9.2 to 246 per million residents a year [1]. Since systematic SCI rehabilitation programs were introduced, mortality rates decreased and life expectancy increased among patients with SCI [2]. However, mortality rates and life expectancy vary in different countries. That is probably due to

Vesna Miloshevska Jakimovska

v.m.jakimovska@studmed.uio.no

1 Sunnaas Rehabilitation Hospital, Nesoddtangen, Norway

2 Faculty of Medicine, University of Oslo, Oslo, Norway

3 Clinic for Spinal Cord Injuries, Rigshospitalet, University of Copenhagen, Copenhagen, Denmark differences in early and long-term evaluation and treatment, employment, mobility aids, social and financial support, etc. The Former Yugoslav Republic of Macedonia (FYROM) has a population of $\sim 2$ million on an area of $25713 \mathrm{~km}^{2}$. The University Clinic for Traumatology, Orthopedics, Anesthesia, Reanimation, Intensive Care Unit and Emergency Center (TOARILUC), Mother Teresa, Skopje, FYROM, is a level one trauma hospital and the only public hospital treating patients with SCI in the country. In recent years, private hospitals also treating persons with SCI have been established in Skopje, but due to very high expenses, these services are out of reach for most citizens of FYROM. To our knowledge, there are no published studies on epidemiology, early evaluation and treatment of patients with SCI in FYROM. The purpose of this study was to prospectively investigate incidence, cause of injury, demography and SCI characteristics, acute phase mortality/ survival, and early complications after tSCI among patients 
admitted to the University Clinic for TOARILUC. The findings may point to issues relevant for the prevention of tSCI in FYROM and also indicate problem areas that need to be improved in the acute care after SCI.

\section{Methods}

Patients with acute tSCI, who were citizens of FYROM and admitted to TOARILUC, Skopje, FYROM in the period from March 1st 2015 to August 31th 2016 (18 months) were invited to participate in the study. If vital signs were stable, an extensive examination was done within the first 6 $\mathrm{h}$ after the injury, including: the International Standards for Neurological Classification of SCI (ISNCSCI), if the patient was conscious and awake [3], Glasgow Coma Scale (GCS), Computerized tomography (CT) scan, and Magnetic resonance imaging (MRI) of the spine. Patients were interviewed daily and/or information was extracted from the patient's medical record until discharge. The information included: demographic data (gender, age at injury, marital status, and employment status), past illnesses, addiction habits (smoking, drugs, and alcohol consumption), preadmission records from patients transported from other hospitals, type of transport to the hospital, date of admission and discharge from TOARILUC, cause and type of injury, associated injuries, and mortality. Selected parts of the following International SCI Data Sets were used to retrieve the specified information mentioned above: International SCI Core Data Set $[4,5]$, International SCI Spinal Column Injury Basic Data Set [6], International SCI Spinal Interventions and Surgical Procedures Basic Data Set [7], and the International SCI Skin and Thermoregulation Function Basic Data [8, 9]. All questionnaires, were translated from English to Macedonian by two independent translators, according to the International Spinal Cord Society recommendations [10].

The initial evaluation and diagnosis were performed in the Emergency Center (EC). All changes in medical condition were recorded on daily basis until death or discharge from the trauma ward or from the intensive care unit (ICU). Body temperature was measured daily for all participants (forehead and axillary). Cultures were grown weekly from the upper respiratory tract from patients on mechanical ventilation. Culture from the wounds, blood, and urine were taken if there was clinical indication of infection or sepsis.

Patients who were discharged or died at the day of admission were categorized as 1 day stay. A 1 year inclusion period was defined as from March 1st 2015 to February 29th 2016. Four seasons were defined as follows: winter (1st December to 28th February), spring (1st March to 31st May), summer (1st June to 31st august), and autumn (1st September to 30th November). We defined a complication as a secondary condition that occurred during the hospital stay because of the current trauma. We defined "polytrauma" as injury to at least two different organ systems of which at least one is life-threatening. A life-threatening injury [11] was defined as an injury that could cause death without emergency assistance. Associated injuries were defined as injuries that occurred at the same time as the SCI, and due to the current trauma. Associated injuries included moderate to severe traumatic brain injury, nonvertebral fractures requiring surgery among more, according to International SCI Core Data Set [4]. Surgical wound infection was suspected when an area (at the incision site) showed signs of rubor, oedema, pain and increased temperature with or without secretion, or a wound not healing as expected. Signs suggestive of an urinary tract infection was malodorous and cloudy urine, muscular spasticity, fatigue, fevers, chills, and autonomic instability. Sepsis was suspected by the presence of two or more of the following: abnormal body temperature, increased heart rate above 100 beats per minute, increased breathing rate above 20 respirations per minute or arterial blood gas analyses and white blood count, confirmed with positive blood culture [12]. Obstipation was defined as reduced frequency or ease of stool passage from what is deemed the normal expected pattern for that individual. Acute diarrhea was defined as the abrupt onset of three or more loose stools per day lasting no longer than 14 days, chronic diarrhea was defined as a period lasting longer than 14 days. Melena was suspected by the passage of dark tarry stools containing decomposing blood, the cause of melena was confirmed by gastroscopy.

We present the patients, following the most recent recommendations [5, 13], in five neurological subgroups according to the neurological level of injury (NLI) and the American Spinal Injury Association (ASIA) Impairment Scale (AIS) grade: I (C4 AIS ABC), II (C5-C8 AIS ABC), III (Th1-S5 AIS ABC), IV (AIS D any NLI), and V (ventilator-dependent any NLI and AIS grade). Adhering to the same recommendations, the following age groups were used: $16-30$ years, $31-45$ years, $46-60$ years, $61-75$ years, and $76+$ years. Patients (or closest relative) gave written informed consent, in accordance with the Declaration of Helsinki. The study was approved by the Director of Department for Traumatology at TOARILUC and by the Norwegian regional ethical committee (REK-Helse Sor OST: 2014/2041/REK South East A).

\section{Statistical analyses}

The statistical analyses were performed with SPSS version 25 (Chicago, IL). Descriptive statistics include range, median, mean, and standard deviation. 


\section{Results}

\section{Epidemiology of SCI}

\section{Demography and incidence}

Thirty-eight patients were included during the 18 months period. The annual incidence was 13 persons with tSCI/ million inhabitants per year. See Table 1 for description of the participants. The total number of patients with spinal column fractures admitted during the 18 months inclusion period was 149 patients. For the 38 participants, mean age at injury was 43 , median was 41 years (range 17-83), and

Table 1 Demographic characteristics of the participants

\begin{tabular}{|c|c|c|}
\hline Man/woman $(n-38)$ & & $32 / 6$ \\
\hline \multicolumn{3}{|l|}{ Age groups } \\
\hline $17-30$ & & 12 \\
\hline $31-45$ & & 8 \\
\hline $46-60$ & & 8 \\
\hline $61-75$ & & 4 \\
\hline 76 and above & & 3 \\
\hline Missing age & & 3 \\
\hline \multicolumn{3}{|l|}{ Etiology } \\
\hline Sport & & 3 \\
\hline Assault & & 1 \\
\hline Traffic accidents & & 16 \\
\hline Car & 11 & \\
\hline Bicycle & 1 & \\
\hline Motorcycle & 2 & \\
\hline Truck & 2 & \\
\hline Falls & & 15 \\
\hline Falls, same level & 5 & \\
\hline Falls from height & 10 & \\
\hline Other & & 2 \\
\hline Unknown & & 1 \\
\hline \multicolumn{3}{|c|}{ NLI and AIS classification } \\
\hline $\mathrm{C} 4 \mathrm{ABC}$ & & 8 \\
\hline $\mathrm{C} 5-\mathrm{C} 8 \mathrm{ABC}$ & & 5 \\
\hline TH1-S5 ABC & & 12 \\
\hline D any level & & 7 \\
\hline Missing & & 6 \\
\hline \multicolumn{3}{|l|}{ Seasons of injury } \\
\hline Winter 20155 & & 5 \\
\hline Spring 2015/2016 & & $7 / 7$ \\
\hline Summer 2015/2016 & & $7 / 6$ \\
\hline Autumn 20156 & & 6 \\
\hline
\end{tabular}

Numbers are absolute

$N L I$ neurological level of injury, AIS American Spinal Injury Association (ASIA) Impairment Scale one third being between 17 and 30 years. The women $(n=$ 6) were all complete injuries and aged between 17 and 45 years. The distribution of tSCI during the four seasons was not significantly different.

\section{Etiology}

The most frequent causes of tSCI were traffic accidents (42\%) followed by falls (40\%) (Table 1). The most frequent traffic accident category included car drivers. Of all fall related injuries $54 \%$ were work related: four construction workers fell from $>8 \mathrm{~m}$ height, one agriculture worker fell from a chestnut tree (around $8 \mathrm{~m}$ ) while picking chestnuts, and two fell from a tractor. There were three described suicide attempts, which all were fall from height. All "sport and leisure injuries" (8\%) were caused from diving into shallow water in rivers. Within the category "other injuries" $(11 \%)$, one construction worker was injured from a crane that fell over him and another was caught beneath a bull, i.e., altogether $24 \%(n=9)$ of all accidents were work related.

Traffic accidents occurred most commonly to patients aged 16-45 years, diving injuries among patients aged 16-30 years, while fall at the same level was the most frequent cause of injury in those aged $60+$ years. Working accidents were most common among construction workers $(n=5)$ and workers in agriculture $(n=4)$, most often seen in patients aged $46-60$ years.

The number of years of education among the participants was as follows: 8 years (18\%), 12 years $(29 \%)$, above 12 years (11\%), and unknown (29\%). Employment status was as follows: unemployed $16 \%$, employed $40 \%$, pensioners $8 \%$, volunteer $3 \%$, and unknown $33 \%$.

Five participants had preexisting diabetes mellitus, one had Bechterew's disease, one had cholelithiasis, three were diagnosed with psychiatric or addiction related diagnosis and those were the same patients that attempted suicide.

\section{Neurological level and extent of SCl}

Twenty-five patients were classified by $\mathrm{CT}$ and MRI as having a cervical-, six a thoracic-, and seven a lumbar-level of SCI. Seventeen patients had a complete (AIS A) SCI and 15 an incomplete neurological injury (see Table 1), six were not available for NLI and AIS classification. Out of these six patients, two died shortly after the arrival at the hospital, and two were in coma until they died. The last two patients were not stable on admission and later they could not be contacted due to restriction upheld from the police force. Of the 16 ventilator-dependent patients, nine had a polytrauma and ten had a cervical SCI. Eleven patients had a spinal column fracture and spondylolisthesis, eight patients did not have a spinal column fracture; six had cervical traumatic 
spondylolisthesis and two spinal cord contusion (cervical level). Two patients with a spinal column fracture had hematomyelia (both cervical level).

\section{Transport to the Emergency Center of TOARILUC}

Thirteen patients with SCI from Skopje were transported directly from the scene of accident with ambulance to the EC of TOARILUC, 23 patients were transported from other hospitals within hours after the trauma, and two patients were transported from other hospitals after 2 days of hospitalization. All patients had cervical collar and were on spine board when they arrived in the EC.

\section{Acute medical care at the EC of TOARILUC}

For all admitted patients in the EC, Glasgow coma score and ISNCSCI were performed if vital signs were stable and the patient were awake and conscious. All patients were examined with CT scan of the head, cervical spine, thorax, abdomen, and pelvis. If diagnosed with an SCI, MRI of the spinal cord at the injured level was performed immediately after the patient was hemodynamically stable. Spinal surgery with decompression of the spinal cord and spinal stabilization was performed as soon as possible when the condition of the patient allowed it. Twenty-two of the patients were operated within the first $24 \mathrm{~h}$ after admission, 11 were not operated as these patients were considered critically unstable, one participant died within $4 \mathrm{~h}$ after admission, and four were diagnosed with contusion $(n=2)$ or hematomyelia $(n=2)$ and were not operated.

\section{Length of stay}

Hospital length of stay ranged from 1 to 73 days, with a median of 20.5 days (Table 2). Patients developing complications were at ICU for more than 30 days.

\section{Early complications after $\mathrm{SCl}$}

\section{Associated injuries}

Twenty patients (53\%) had a polytrauma. The associated injuries included fractures of the long bone of the lower extremities $(n=3)$, pelvis $(n=3)$ and upper extremities $(n$ $=4)$, thoracic $(n=18 ; 11$ pulmonary contusions, five pneumothorax, six hematothorax, seven with rib fractures, and one sternal fracture) and abdominal injuries $(n=4$; three retroperitoneal bleedings and one lineal laceration), and head trauma $(n=11)$.

At admission $29 \%$ were diagnosed with a head trauma, five were scored with a GCS above 8 and $<15$, and six persons were scored with an GCS $<8$. The total number of participants with a severe brain injury was six; of these, four developed brain hemorrhages (two operated immediately), two were diagnosed with fractures of the facial bones, two were diagnosed with a skull fracture, and two with an edema of the brain. At discharge, all surviving patients had a GCS above 12 .

Lower extremities were operated with proximal femoral nail and K-wire fixation (metatarsal bone fracture). Pleural complications were treated with thoracic drainage immediately on admission, one patient had an additional decortication of the pleura. One patient underwent an explorative laparotomy with a suture of a ventricular ulcer because of an ongoing bleeding.

\section{Complications during hospital stay}

Fifteen patients suffered a total of 27 complications (see Table 3). The most common complications were pressure ulcers $(n=8)$, gastrointestinal related $(n=8)$, and infections $(n=7)$. All patients with pressure ulcers were hospitalized for a period between 20 and 77 days, of these five were on mechanical ventilation for 37-55 days. Pressure ulcers were localized on both heels in seven patients, three patients had additional pressure ulcers on the sacrum. One patient had pressure ulcers on both malleoli and on the sacrum. No pressure ulcer extended through subcutis and none were surgically reconstructed during the hospitalization. Out of the six women, four were on ventilator, and one developed pressure ulcer. At discharge, the same seven participants had pressure ulcers, category II or III [9]. Gastrointestinal complications included melena $(n=4)$, diarrhea $(n=1)$, and obstipation $(n=2)$. One participant developed melena and diarrhea. Infections occurred between the 10th and the 20th day of hospitalization. We observed, most commonly, pulmonary-related infections and only one urinary tract infection. Cultures from the intubation tubes (or from the tracheostoma) revealed Pseudomonas aeruginosa, Klebsiella, Methicillin Resistant Staphylococcus Aureus (MRSA), and Acinetobacter species in seven patients. Blood culture was MRSA positive in two patients. Urine culture was Acinetobacter positive in one patient. There was only one surgical wound infection (colonized with Klebsiella). There were no correlations between complications and prior history of diabetes, smoking, or alcohol consumption. Of the patients with previous morbidities, one patient developed melena.

\section{Mortality}

Mortality cases are described in Table 4. Twelve (32\%) patients died between 1 and 56 days, median 22.5 days. Their age ranged from 17 to 83 years, median $49(50( \pm 19))$ 
Table 2 Length of stay

\begin{tabular}{lllll}
\hline Total & $\begin{array}{l}\text { Up to 2 days } \\
n=6(16 \%)\end{array}$ & $\begin{array}{l}3-15 \text { days } \\
n=8(21 \%)\end{array}$ & $\begin{array}{l}16-30 \text { days } \\
n=12(32 \%)\end{array}$ & $\begin{array}{l}\text { More than 30 days } \\
n=10(26 \%)\end{array}$ \\
\hline $\begin{array}{l}\text { Politrauma }(n=20) \\
\text { Died in hospital }(n=12)\end{array}$ & $2(10 \%)$ & $5(25 \%)$ & $7(35 \%)$ & $6(30 \%)$ \\
NLI and AIS classification & $3(25 \%)$ & $3(25 \%)$ & $2(17 \%)$ & $4(33 \%)$ \\
C4 AIS ABC $(n=9)$ & $3(33 \%)$ & $2(22 \%)$ & $1(11 \%)$ & $3(33 \%)$ \\
C5-C8 AIS ABC $(n=5)$ & & $1(20 \%)$ & $1(20 \%)$ & $3(60 \%)$ \\
Th1-S5 AIS ABC $(n=12)$ & & $2(17 \%)$ & $6(50 \%)$ & $4(33 \%)$ \\
DE AIS Any level $(n=7)$ & & $3(43 \%)$ & $4(57 \%)$ & \\
\hline
\end{tabular}

Numbers are absolute or \% calculated within rows

$N L I$ neurological level of injury, AIS American Spinal Injury Association (ASIA) Impairment Scale

Table 3 Difference in distribution of complications between groups

\begin{tabular}{|c|c|c|c|c|c|c|c|c|}
\hline Complication & $\begin{array}{l}\text { Pulmonary } \\
\text { infection }\end{array}$ & $\begin{array}{l}\text { Sepsis } \\
\text { infection }\end{array}$ & $\begin{array}{l}\text { Wound } \\
\text { infection }\end{array}$ & $\begin{array}{l}\text { Urinary } \\
\text { infection }\end{array}$ & Pressure ulcers & Melena & Obstipation & Diarrhea \\
\hline \multicolumn{9}{|l|}{$\begin{array}{l}\text { NLI and AIS } \\
\text { classification }\end{array}$} \\
\hline $\mathrm{C} 4 \mathrm{ABC}$ & $1^{*}$ & & & $1^{*}$ & $2 *$ & & & \\
\hline C5-C8 ABC & $1^{*}$ & & & & $1 *$ & $1^{*}$ & & \\
\hline Th1-S5 ABC & $3^{*}$ & $1^{*}$ & & & $4(2 *)$ & $2(1 *)$ & 2 & \\
\hline D any level & 1 & $1^{*}$ & $1^{*}$ & & 1 & $2(1 *)$ & 1 & 1 \\
\hline Total & 6 & 2 & 1 & 1 & 8 & 5 & 3 & 1 \\
\hline
\end{tabular}

Numbers are absolute numbers

$N L I$ neurological level of injury, AIS American Spinal Injury Association (ASIA) Impairment Scale

Asterisk indicates ventilator dependent patients

years. The participants in the groups above 61 years of age, had a $90 \%$ mortality, the mortality was reduced by $25 \%$ for each age-group in order of declining age. Seven of the deceased were classified as $\mathrm{C} 4$ AIS ABC, five were classified as Th1-S5 AIS ABC. Fall from height was the cause of injury among $41 \%$ of the deceased. Among the initially ventilator-dependent patients $(n=16), 50 \%$ had a lethal outcome.

\section{Discharge}

The survivors were discharged either to their homes $(n=$ $15)$, to a rehabilitation (public) hospital $(n=5)$ or a private hospital $(n=5)$. Of those transferred to a public rehabilitation hospital, two were discharged to a public psychiatric hospital and one to a geriatric department. Five patients were transferred (on their demand) to a private hospital. Of these, two were transferred at the day of admission to a private hospital, one was later transferred back to TORILIUC due to the patients financial challenges related to the private hospital expenses. All patients with AIS ABC were discharged with indwelling catheter and incontinence equipment such as diapers.

\section{Discussion}

We here present epidemiological data on citizens of FYROM acquiring a tSCI and admitted to TOARILUC from March 1st 2015 to August 31th 2016. We report an incidence of 13 persons with a tSCI per million inhabitants per year. Further, we describe the demographic data, route and LOS in the hospital, past illnesses, injury-related information, type of injury and its level and extent, associated injuries, complications, and mortality in a total of 38 patients.

Our main findings are that the mean age at injury was 43 years (median 41 years, range 17-83) with a male/female ratio of 5 to 1 . Seventeen patients had a complete (AIS A) SCI and 15 an incomplete neurological injury. Most frequent etiology for SCI was traffic accidents (42\%) followed by falls $(40 \%)$. Twenty four percent were accidents at work. Sixteen patients were ventilator-dependent at some point during the acute period. In our study, patients with severe and multiple injuries mostly to the thorax and brain were more prone for complications, especially those who were on mechanical ventilation. Common complications were pressure ulcers, gastrointestinal related (melena, obstipation, 


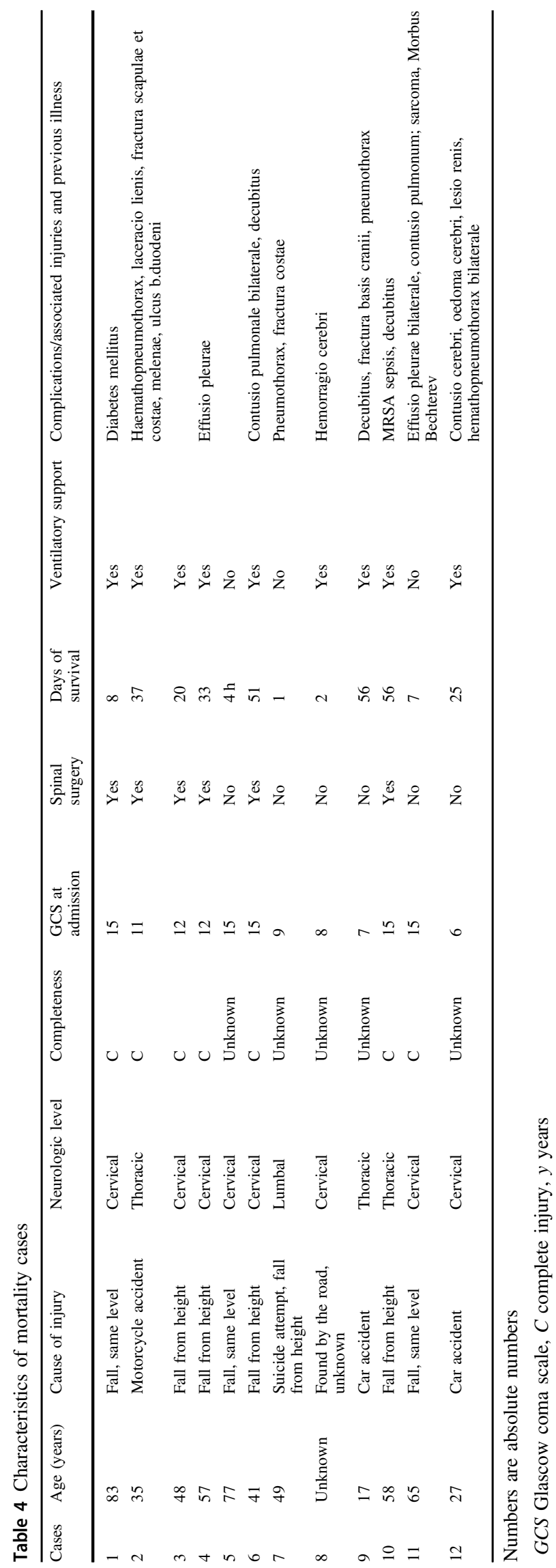

and diarrhea), and infections (most frequent pulmonary related). The mortality was $32 \%$ during the first $2 \frac{1}{2}$ months post injury, and as high as $90 \%$ among patients above 60 years of age.

\section{Incidence}

Studies from regions geographical close to FYROM, such as the Thessaloniki region in Greece, report an incidence of 30 persons/million residents per year [14]. Our lower incidence of 13 persons/million inhabitants per year could in part be due to higher mortality after traffic accidents before diagnosed with a SCI. The high incidence of brain injuries (29\%) and polytrauma among our participants could be an indication of a greater forces involved in the accidents, one could speculate that this underlines our suspicion of higher mortality at the time of injury. Another reason could be that persons with traumatic SCI may have deceased in other local hospitals in FYROM before diagnosed with a SCI.

\section{Demographics}

The male/female ratio was $5: 1$ in our study sample is similarly to findings in studies from developing parts of the world [14]. The male/female ratio is reported to be higher in developing compared with developed countries [15]. There is a general trend in the developed countries of increasing age at SCI, our participants were $\sim 10$ years younger than patients from other European countries such as Finland and Scotland $[16,17]$. The most frequent cause of injury in our study was traffic accidents, which is in line with findings in Greece [14]. Roads and the motor vehicle fleet in FYROM are old and often in bad condition. Preventive measures as enforcement of speed limits, use of safety belts, and regular control of the cars standard must be taken to reduce SCI related to road- and traffic- conditions.

In contrast, falls is the leading cause to SCI in many other European countries [16-18]. In order to be able to develop prevention strategies, it is important to realize the mechanisms of the falls [19]. In our sample, work accidents happened to individuals between 46 and 60 years of age and most frequently among workers at construction sites, and within agriculture. Therefore the use of safety lines and railings at the construction sites need to be enforced like other safety gear such as helmets, shoes, etc. Falls at the same level was the leading cause of SCI for the age group above 60 , a trend similarly observed in developed countries with the aging population [20].

Sport injuries in our data were caused by recreational diving into shallow water of rivers. These were all in rural areas and patients were in their early twenties. Other studies have confirmed diving into shallow water to be the most common sport or leisure time activity resulting in tSCI 
worldwide [21, 22]. Education in schools, and posters at relevant places with warnings are examples of prevention measures that could help reduce diving related SCI in the future.

\section{Complications and associated injuries}

Complications after tSCI occurred in $39 \%$ of the patients. It is estimated that patients with tSCI have injuries to other organ systems in $80 \%$ of the cases [23]. In our study more than $90 \%$ of the complications were in patients with a polytrauma and most of them were on ventilator support. In the Thessaloniki study [14, 24], life threatening injuries was observed in $23 \%$ of the patients, while in our study $50 \%$ of the patients had life threatening injuries.

Pressure ulcers were one of the most prevalent complications in our patients, which is in accordance with other studies $[25,26]$. However, our data of $21 \%$ of patients with pressure ulcers is low compared with other reports [25, 26]. We assume this is because our hospital has a long experience with critical ill patients and precautions were taken together with the use of antidecubital mattresses, to avoid pressure ulcers. We observed no association between NLI and pressure ulcers, which likewise was experienced by others [27]. Infections were common, as reported in other studies [28-30]. We observed that participants with more than 2 ICU days and between 10 and 20 ventilator days more frequently developed ventilator associated pneumonia, which also has been observed previously [31]. We have not observed other complications which are otherwise commonly reported, such as deep vein thrombosis and pulmonary embolism [32-34], the patients were under constant monitoring for changes in hemostasis and the hospital use compression stockings and Clexane $(40 \mathrm{mg} \times 1$ s.c, continued up to 6 weeks). Furthermore, we did not observe instances of autonomic dysreflexia, a possible cause could be a high mortality rate among cervical complete SCI patients and short LOS. We registered only one urinary tract infection, we assume the possible reason is the frequent use of long-term antibiotic treatment during their stay at the hospital.

\section{Mortality}

We report a high inhospital mortality rate (32\%). Our mortality rate during the observation period of 2.5 months after injury exceeds even that of the first year mortality rate in neighboring countries. First year mortality rate after tSCI ranges from $0.6 \%$ in Turkey to $20 \%$ in Finland $[1,35]$. In Thessaloniki the first year mortality rate was reported to be $20 \%$ [36], however they have excluded patients that died the first week of the injury. In our study, $75 \%$ of those who died in hospital were on ventilator support. Previous studies have shown that persons with SCI in the need of mechanical ventilator support have a reduced survival rate [34]. We reported $90 \%$ mortality rate among elderly (above 60 years of age) in line with other reports [35]. We had one patient with Bechterew's disease who died after 1 week at the ICU, and as shown previously [37] these patients are at higher risk of early death after SCI than those without this spinal condition. All in all the injuries reported in this study seem to be more severe than reported from other countries and hospitals, furthermore a combination of severe injuries (polytrauma, including severe head trauma) and complications, most likely contribute to the high inhospital mortality. The hospital personnel is highly educated and experienced in treating polytraumatised, still prevention is possible only if the reason for death is known. Autopsies are not routinely performed because of cultural believes in our country, although according to the law every death in a hospital should be further examined with an autopsy.

\section{Discharge}

Acute care for patients with SCI in FYROM is mainly centralized to TOARILUC. Specialized SCI rehabilitation centers are nonexisting. The patients are discharged to home before being admitted to different rehabilitation hospitals across the country, with different levels of experience and expertise. No other SCI related follow up routines are established in FYROM.

\section{Strengths and Limitations}

The strengths of our study are that in FYROM, TOARILUC is the only public hospital treating tSCI. The first author contacted colleagues, orthopedic surgeons, at Orthopedic hospital St.Erasmo-Ohrid (they treat scoliosis and vertebral fractures/dislocations without SCI), and the other hospital that has orthopedic ward in Skopje, named 8th of September, and they both confirmed that they do not treat SCI patients. This information is not official and is not in a written form. There was an agreement many years ago that the Spine Unit at TOARILUC will be the only hospital to treat tSCI in North Macedonia. The National Statistical Office was contacted as well, but they do not hold information related to the organization of the acute care of tSCI. In addition, there is an agreement that all polytraumatized patients to be admitted to TOARILUC. The authors cannot know if everyone adhere to this agreement. In recent years, private hospitals have been established in Skopje that are able to treat patients with SCI, but the cost is very high, thus excluding most citizens. To the best of our knowledge, private hospitals received acute patients with tSCI from Kosovo mainly, in addition to the five patients transferred from our hospital. A further advantage for the study is that 
all but one of the ISNCSCI assessments, all the clinical examinations and interviews were carried out by one person only (the first author) an experienced clinician with proper training in ISNCSCI, thus all the data are collected and analyzed alike.

A limitation of our study is the small number of participants. Nevertheless, our observations seem important for the direction of implementation of preventing measures. Deaths that occurred at the scene of accidents were not reported, and thus the yearly incidence of traumatic SCI may be underreported. As autopsies were not performed, the cause of death at the hospital is uncertain. The disadvantages of direct recruitment by one research personnel could be associated with risk of biased recruitment, exhaustion of the examiner and not having a second opinion available.

\section{Conclusion}

We have outlined the tSCI patient's way from the scene of accident throughout the acute phase to the rehabilitation phase in FYROM. Due to high mortality and in-hospital complications, even though common complications like autonomic dysreflexia were not observed, we believe this study can raise awareness of their existence and help improve care of future patients. In addition, passive and lung physiotherapy is not established to the point of accomplishing success for all patients at ICU because of lack of staff. Therefore this is another important message from this study and could be an area for future intervention and research. Furthermore, there is no centralized rehabilitation center for SCI persons after they leave the acute clinic. To be educated about their new living condition and precaution measures for the skin, urinary tract etc., is a very important part of the comprehensive SCI treatment. Our findings indicate that preventive strategies should focus on traffic-, fall related-, and diving accidents, including the work related accidents. There is a relatively low yearly incidence of $13 \mathrm{SCI} / \mathrm{million}$ inhabitants, probably due to underreported deaths due to SCI at the scene of accident or at other local hospitals. We found a high in-hospital mortality rate of $32 \%$, most likely due to the high degree of polytrauma among our patients. We observed that patients with extended stay in ICU developed more complications. Finally, the study points to the need for organized rehabilitation for SCI patients after discharge from the acute hospital.

\section{Data archiving}

The data sets generated and analyzed during the current study are available from the corresponding author on reasonable request.
Acknowledgements We want to thank the study participants, the Sunnaas Rehabilitation Hospital, and the QUOTA program for contributing with financial support to the project.

Funding The study was supported by the Quota program, Norway.

Author contributions $\mathrm{VJ}$ was responsible for designing and writing the protocol, inclusion of participants, extracting and analysing data, interpreting results, creating the reference lists and tables, and writing the paper. FBS contributed to designing and writing the protocol, extracting and analysing data interpreting results, and providing feedback on the paper. IBL contributed to designing and writing the protocol, extracting and analysing data, interpreting results, and providing feedback on the paper. EK contributed to designing and writing the protocol, inclusion of participants, extracting and analysing data, interpreting results, and providing feedback on the paper.

\section{Compliance with ethical standards}

Conflict of interest The authors declare that they have no conflict of interest.

Ethics statement We certify that all applicable institutional and governmental regulations concerning the ethical use of human volunteers were followed during the course of this research.

Publisher's note: Springer Nature remains neutral with regard to jurisdictional claims in published maps and institutional affiliations.

\section{References}

1. Jazayeri SB, Beygi S, Shokraneh F, Hagen EM, RahimiMovaghar V. Incidence of traumatic spinal cord injury worldwide: a systematic review. Eur Spine J. 2015;24:905-18.

2. DeVivo MJ, Krause JS, Lammertse DP. Recent trends in mortality and causes of death among persons with spinal cord injury. Arch Phys Med Rehabil. 1999;80:1411-9.

3. Kirshblum SC, Burns SP, Biering-Sorensen F, Donovan W, Graves DE, Jha A, et al. International standards for neurological classification of spinal cord injury (revised 2011). J Spinal Cord Med. 2011;34:535-46.

4. DeVivo M, Biering-Sorensen F, Charlifue S, Noonan V, Post M, Stripling T, et al. International Spinal Cord Injury Core Data Set. Spinal Cord. 2006;44:535-40.

5. DeVivo MJ, Biering-Sorensen F, New P, Chen Y. Standardization of data analysis and reporting of results from the International Spinal Cord Injury Core Data Set. Spinal Cord. 2011;49:596-9.

6. Dvorak MF, Wing PC, Fehlings MG, Vaccaro AR, Itshayek E, Biering-Sorensen $\mathrm{F}$, et al. International spinal cord injury spinal column injury basic data set. Spinal Cord. 2012;50:817-21.

7. Dvorak MF, Itshayek E, Fehlings MG, Vaccaro AR, Wing PC, Biering-Sorensen $\mathrm{F}$, et al. International spinal cord injury: spinal interventions and surgical procedures basic data set. Spinal Cord. 2015;53:155-65.

8. Karlsson AK, Krassioukov A, Alexander MS, Donovan W, Biering-Sorensen F. International spinal cord injury skin and thermoregulation function basic data set. Spinal Cord. 2012;50:512-6.

9. Biering-Sorensen F, Alexander MS, van Asbeck FWA, Donovan W, Krassioukov A, Post MWM. Version 1.1 of the international spinal cord injury skin and thermoregulation function basic data set. Spinal Cord. 2017;55:566-9. 
10. Biering-Sorensen F, Alexander MS, Burns S, Charlifue S, DeVivo M, Dietz V, et al. Recommendations for translation and reliability testing of International Spinal Cord Injury Data Sets. Spinal Cord. 2011;49:357-60.

11. Fedakar R, Aydiner AH, Ercan I. A comparison of "life threatening injury" concept in the Turkish Penal Code and trauma scoring systems. Ulus Travma Acil Cerrahi Derg. 2007; 13:192-8

12. Singer M, Deutschman CS, Seymour CW, Shankar-Hari M, Annane D, Bauer M, et al. The Third International Consensus Definitions for Sepsis and Septic Shock (Sepsis-3). JAMA. 2016;315:801-10.

13. Biering-Sorensen F, DeVivo MJ, Charlifue S, Chen Y, New PW, Noonan V, et al. International Spinal Cord Injury Core Data Set (version 2.0)-including standardization of reporting. Spinal Cord. 2017;55:759-64.

14. Divanoglou A, Levi R. Incidence of traumatic spinal cord injury in Thessaloniki, Greece and Stockholm, Sweden: a prospective population-based study. Spinal Cord. 2009;47:796-801.

15. Moshi H, Sundelin G, Sahlen KG, Sorlin A. Traumatic spinal cord injury in the north-east Tanzania-describing incidence, etiology and clinical outcomes retrospectively. Glob Health Action. 2017;10:1355604.

16. McCaughey EJ, Purcell M, McLean AN, Fraser MH, Bewick A, Borotkanics RJ, et al. Changing demographics of spinal cord injury over a 20-year period: a longitudinal population-based study in Scotland. Spinal Cord. 2016;54:270-6.

17. Niemi-Nikkola V, Saijets N, Ylipoussu H, Kinnunen P, Pesala J, Makela $\mathrm{P}$, et al. Traumatic Spinal Injuries in Northern Finland. Spine. 2018;43:E45-E51.

18. Ferro S, Cecconi L, Bonavita J, Pagliacci MC, Biggeri A, Franceschini M. Incidence of traumatic spinal cord injury in Italy during 2013-2014: a population-based study. Spinal Cord. 2017;55:1103-7.

19. Chen Y, Tang Y, Allen V, DeVivo MJ. Fall-induced spinal cord injury: external causes and implications for prevention. J Spinal Cord Med. 2016;39:24-31.

20. Zhou Y, Wang XB, Kan SL, Ning GZ, Li YL, Yang B, et al. Traumatic spinal cord injury in Tianjin, China: a single-center report of 354 cases. Spinal Cord. 2016;54:670-4.

21. Chan CWL, Eng JJ, Tator CH, Krassioukov A. Epidemiology of sport-related spinal cord injuries: a systematic review. J Spinal Cord Med. 2016;39:255-64.

22. Barss P, Djerrari H, Leduc BE, Lepage Y, Dionne CE. Risk factors and prevention for spinal cord injury from diving in swimming pools and natural sites in Quebec, Canada: a 44-year study. Accid Anal Prev. 2008;40:787-97.

23. Yue JK, Winkler EA, Rick JW, Deng H, Partow CP, Upadhyayula PS, et al. Update on critical care for acute spinal cord injury in the setting of polytrauma. Neurosurg focus. 2017;43:E19.
24. Divanoglou A, Seiger A, Levi R. Acute management of traumatic spinal cord injury in a Greek and a Swedish region: a prospective, population-based study. Spinal Cord. 2010;48:477-82.

25. Joseph C, Nilsson Wikmar L. Prevalence of secondary medical complications and risk factors for pressure ulcers after traumatic spinal cord injury during acute care in South Africa. Spinal Cord. 2016;54:535-9.

26. Scheel-Sailer A, Wyss A, Boldt C, Post MW, Lay V. Prevalence, location, grade of pressure ulcers and association with specific patient characteristics in adult spinal cord injury patients during the hospital stay: a prospective cohort study. Spinal Cord. 2013;51:828-33.

27. Gelis A, Dupeyron A, Legros P, Benaim C, Pelissier J, Fattal C. Pressure ulcer risk factors in persons with SCI: Part I: acute and rehabilitation stages. Spinal Cord. 2009;47:99-107.

28. Nicolle LE. Urinary tract infections in special populations: diabetes, renal transplant, HIV infection, and spinal cord injury. Infect Dis Clin North Am. 2014;28:91-104.

29. Haisma JA, van der Woude LH, Stam HJ, Bergen MP, Sluis TA, Post MW, et al. Complications following spinal cord injury: occurrence and risk factors in a longitudinal study during and after inpatient rehabilitation. J Rehabil Med. 2007;39:393-8.

30. Aito $\mathrm{S}$. Complications during the acute phase of traumatic spinal cord lesions. Spinal Cord. 2003;41:629-35.

31. Michetti CP, Prentice HA, Rodriguez J, Newcomb A. Supine position and nonmodifiable risk factors for ventilator-associated pneumonia in trauma patients. Am J Surg. 2017;213:405-12.

32. Marion TE, Rivers CS, Kurban D, Cheng CL, Fallah N, Batke J, et al. Previously identified common post-injury adverse events in traumatic spinal cord injury-validation of existing literature and relation to selected potentially modifiable comorbidities: a prospective Canadian cohort study. J Neurotrauma. 2017;34: 2883-91.

33. Hagen EM. Acute complications of spinal cord injuries. World $\mathbf{J}$ Orthop. 2015;6:17-23.

34. Jia X, Kowalski RG, Sciubba DM, Geocadin RG. Critical care of traumatic spinal cord injury. J Intensive Care Med. 2013;28: $12-23$.

35. Majdan M, Plancikova D, Nemcovska E, Krajcovicova L, Brazinova A, Rusnak M. Mortality due to traumatic spinal cord injuries in Europe: a cross-sectional and pooled analysis of population-wide data from 22 countries. Scand J Trauma Resusc Emerg Med. 2017;25:64.

36. Divanoglou A, Westgren N, Seiger A, Hulting C, Levi R. Late mortality during the first year after acute traumatic spinal cord injury: a prospective, population-based study. J Spinal Cord Med. 2010;33:117-27.

37. Teunissen FR, Verbeek BM, Cha TD, Schwab JH. Spinal cord injury after traumatic spine fracture in patients with ankylosing spinal disorders. J Neurosurg Spine. 2017;27:709-16. 